\title{
Side Effects of Bone-Targeted Therapies in Advanced Breast Cancer
}

\author{
Christoph Domschke Florian Schuetz \\ Department of Gynecology and Obstetrics, Heidelberg University Hospital, Heidelberg, Germany
}

\section{Keywords}

Breast cancer - Bone-targeted agents - Bisphosphonates . Denosumab - Adverse events

\section{Summary}

In up to $75 \%$ of cases, advanced breast cancer patients eventually develop bone metastases with often debilitating skeletal-related events (SREs). Osteoclast inhibitors are commonly used as therapeutic mainstay with clinical studies showing superiority of denosumab over bisphosphonates (e.g., zoledronate) for the prevention of SREs. The present review discusses the adverse event profile of these agents, and addresses the prevention and management of untoward side effects. Adverse events associated with osteoclast inhibitors comprise osteonecrosis of the jaw and hypocalcemia. Hypocalcemia is more common with denosumab, particularly in severe renal dysfunction. During therapy, the appropriate prevention of these adverse events includes close attention to dental health, avoidance of invasive dental procedures, supplementation with calcium and vitamin D unless patients are hypercalcemic, and regular monitoring of relevant serum values. Relating to the risk of nephrotoxicity, bisphosphonates but not denosumab have been incriminated. Therefore, serum creatinine levels should be checked prior to each dose of zoledronate, and in severe renal dysfunction (creatinine clearance $<30 \mathrm{ml} / \mathrm{min}$ ) zoledronate is contraindicated anyway. Acute-phase reactions are particularly linked to bisphosphonates. Consequently, if these adverse events predominate, switching to denosumab is recommended.

\section{Introduction}

In the advanced stage of breast cancer, $65-75 \%$ of patients eventually develop bone metastases $[1,2]$ which often lead to serious and severely debilitating consequences called skeletalrelated events (SREs) including pathologic fractures, spinal cord compression, or the need for palliative irradiation or orthopedic surgery. These complications result in poor quality of life due to massive bone pain and functional disability up to loss of autonomy, and finally in decreased patient survival. It goes without saying that these events also entail a significant economic burden [3-6].

Consequently, it is of paramount importance for the patient to prevent SREs in order to sustain functionality, alleviate pain, improve quality of life, and in the end to further prolong survival. For that purpose, besides various local and systemic therapies including radiation, chemotherapeutics and surgical approaches $[7,8]$, the use of bone-targeted osteoclast inhibitors has led to significant advances $[9,10]$. Osteoclast inhibitors comprise 2 classes of agents, the bisphosphonates and the fully human monoclonal antibody denosumab. Bisphosphonates are chemically related to inorganic pyrophosphate, and are particularly bone-active agents as they are preferentially adsorbed onto hydroxyapatite crystals in the extracellular matrix of bone. From there, bisphosphonates are taken up by osteoclasts and induce apoptotic processes [11, 12]. Thirdgeneration nitrogen-containing bisphosphonates, such as zoledronic acid, are much more potent than earlier compounds [13]. On the other hand, denosumab binds to the receptor activator of nuclear factor $\mathrm{kB}$ ligand (RANKL) which then binds to its receptor, RANK, located on the osteoclast cell surface, thus interfering with normal osteoclast activation and leading to decreased bone-destructive processes [14, 15].

Despite the overall beneficial effects of osteoclast inhibitors, these therapies, like any medication, are not without un-

\section{KARGER \\ Fax +497614520714 \\ Information@Karger.com}

www.karger.com (c) 2014 S. Karger GmbH, Freiburg

$1661-3791 / 14 / 0095-0332 \$ 39.50 / 0$

Accessible online at:

www.karger.com/brc 
toward effects. Therefore, to facilitate an adequate medical decision making, we here review the adverse event profile of the 2 classes of bone antiresorptive agents in advanced breast cancer [9, 12, 16-18]. In the adjuvant therapy setting of earlystage breast cancer patients, the incidence of similar side effects appears to be lower due to reduced doses and frequencies of osteoclast inhibitors administered [19].

\section{Side Effects of Osteoclast Inhibitors}

Comparative studies of intravenous zoledronic acid and subcutaneous denosumab in patients with metastatic breast cancer $[20,21]$ and prostate cancer $[22,23]$ reported similar adverse event profiles. Side effects encountered most frequently were acute-phase reactions, nephrotoxicity, hypocalcemia, and osteonecrosis of the jaw (ONJ). These will be discussed below in more detail. Very rare adverse events in the gastrointestinal tract are seen exclusively during oral bisphosphonate treatment. Following bisphosphonates, there have also been some few reports of ocular complications in terms of scleritis, uveitis, and conjunctivitis [11, 24-26]. Occasionally, subcutaneous denosumab may induce injection site reactions and other dermatologic conditions such as dermatitis, eczema, and rashes [18, 20]. Rarely, atypical femur fractures have been associated with bisphosphonate and denosumab therapy [23, 27]. So far, no patients have developed detectable levels of neutralizing antibodies against denosumab [20, 22]. In randomized studies of bisphosphonates and denosumab, severegrade adverse events have occurred with similar frequencies between treatment arms [20,22]. Likewise, treatment discontinuation secondary to serious side effects was generally similar in patients on zoledronic acid and denosumab [22, 28-30].

\section{Acute-Phase Reactions}

Acute-phase reactions are defined as flu-like symptoms including subfebrile temperatures, leukocytosis, chills, flushing, abnormal fatigue, bone pain, arthralgias, and myalgias [31]. In a comparative study, acute-phase reactions during the first 3 days after treatment were seen in about 30 and $10 \%$ of breast cancer patients with zoledronic acid and denosumab, respectively [20]. These responses are induced only by nitrogen-containing bisphosphonates (zoledronate, ibandronate, pamidronate) when being administered intravenously. Most acute-phase reactions typically occur in the first 3 days following initiation of treatment, subside rapidly soon after, and often do not recur upon subsequent treatments. The above side effects, although not life-threatening, may be very unpleasant for the patient, and in rare cases lead to termination of the medication.

\section{Management}

Symptomatic relief is provided by nonsteroidal anti-inflammatory drugs and antipyretics [24, 32].

\section{Nephrotoxicity}

After therapeutic administration, bisphosphonates are either stored in the bones $(30-60 \%)$ or remain unmetabolized and are excreted renally (for further pharmacokinetic data see [33-35]). In the kidneys, bisphosphonates diffuse passively into tubular cells dependent only on serum concentration and protein binding. Consequently, an accumulation of bisphosphonates in the tubular cells can occur with induction of apoptotic processes and development of acute kidney injury [12, 36-38] due to tubular necrosis, which typically results in a gradual increase in serum creatinine over months that is only slowly reversed after drug discontinuation [11, 39-41]. Of the available bisphosphonates, zoledronic acid has been linked to most of the reported episodes of renal failure [24, 42]. Recovery from zoledronic acid-induced acute tubular necrosis is possible but may be protracted [38].

Patients with pamidronate-associated renal toxicity may present with nephrotic syndrome which apparently is not reversible $[11,24,43]$. Histologically, these cases show collapsing focal segmental glomerulosclerosis, acute tubular injury, or minimal change disease without glomerular pathology [43]. Ibandronate seems to be the bisphosphonate least likely to be linked to renal toxicity [44].

In contrast, in comparative clinical trials of advanced breast cancer patients, denosumab has been associated with highly significantly lower renal deterioration rates than zoledronic acid [20]. Notably, denosumab has no recognized effect on renal function. Conversely, pre-existing renal impairment was shown to have no impact on denosumab efficacy either. Accordingly, in patients with renal function ranging from normal to dialysis-dependent kidney failure, no effect of renal function on denosumab pharmacokinetics and pharmacodynamics could be encountered. Therefore, no denosumab dose adjustment based on glomerular filtration rate seems necessary $[18,21,45]$.

\section{Management}

Prior to each dose of zoledronic acid, determination of serum creatinine level is required. Bisphosphonate medication should be stopped if the creatinine level increases by $\geq 0.5 \mathrm{mg} / \mathrm{dl}$ in patients with normal baseline renal function and if the creatinine level increases by $\geq 1.0 \mathrm{mg} / \mathrm{dl}$ in patients with abnormal baseline renal function. Treatment can be resumed if the creatinine level returns to near baseline values [23]. Zoledronic acid should be dose reduced in patients with impaired renal function (estimated creatinine clearance $30-60 \mathrm{ml} / \mathrm{min}$ ), and held for creatinine clearance $<30 \mathrm{ml} / \mathrm{min}$ $[21,46]$.

In the case of pamidronate-associated nephrotic syndrome, no standardized therapy exists. Suggested palliative therapies include corticosteroids and angiotensin-converting enzyme inhibitors [11, 24].

General recommendations to reduce bisphosphonate-induced kidney damage comprise maintenance of adequate hy- 
dration, avoidance of concomitant nephrotoxic agents, and strict adherence to package insert information.

Upon denosumab administration, in patients with severely impaired renal function - creatinine clearance $<30 \mathrm{ml} / \mathrm{min}$ or on dialysis - close monitoring for hypocalcemia and hypophosphatemia is recommended $[21,46]$. Clearly, in this patient cohort the experience is very limited as yet.

\section{Hypocalcemia}

Hypocalcemia is the most common electrolyte abnormality associated with the use of bisphosphonates and denosumab $[24,47]$, as this is due to the mechanism of action of antiresorptive agents. In comparative studies, hypocalcemia was documented significantly more frequently with denosumab (5.5-13\% of cases) than with zoledronic acid (3.4-6\%) [20, 22]. Clinically, hypocalcemic patients may appear lethargic or weak or they may have full-blown tetany $[11,18]$. Even fatal cases of denosumab-induced hypocalcemia have been reported, making clear the critical need for adequate calcium and vitamin D supplementation. In a recent trial, the incidence of hypocalcemia due to denosumab was lower in patients who reported taking calcium and vitamin D supplements than among the patients who did not [48]. The risk appears to be greatest in patients with pre-existing hypocalcemia due to impaired thyroid or parathyroid function. The risk of hypocalcemia with denosumab seems also markedly enhanced in patients with renal insufficiency (creatinine clearance $<30$ $\mathrm{ml} / \mathrm{min}$ ) [45] and those with extensive osteoblastic bone metastases or hypomagnesemia [49], as well as after bariatric surgery [50]. The onset of hypocalcemia can arise at any time during therapy, and most commonly occurs within the first 6 months of dosing [21].

\section{Management}

To avoid the occurrence of hypocalcemia in patients prescribed denosumab or bisphosphonates, serum vitamin D3 and albumin-corrected or ionized calcium levels should be monitored before the first injection/infusion and all subsequent therapies so that hypocalcemia at any point during treatment can be identified and corrected [24, 47, 50]. As the normal conversion of vitamin $\mathrm{D}$ to the active form (25-hydroxycholecalciferol to 1.25-hydroxycholecalciferol (calcitriol)), which occurs in the kidneys, is compromised in the condition of renal dysfunction, supplementation with calcitriol has been suggested for the prevention or treatment of hypocalcemia [51].

Breast cancer patients going to be treated with bisphosphonates or denosumab should be normocalcemic at initiation of therapy and then should be regularly supplemented with calcium $(1,000 \mathrm{mg} /$ day orally $)$ plus calcitriol $(0.25 \mu \mathrm{g} /$ day orally $)$. If nevertheless hypocalcemia develops, the osteoclast inhibitor should be held, and calcium and calcitriol doses should be adequately adjusted $[23,24,50]$.

\section{Osteonecrosis of the Jaw}

ONJ is a well-recognized untoward effect associated with the use of both bisphosphonates and denosumab, and comprises osteonecrosis of the mandible and/or maxilla. According to the American Association of Oral and Maxillofacial Surgeons [52], ONJ has been defined as i) the presence of clinically evident necrotic bone exposed through the oral mucosa or facial skin which has persisted despite appropriate management for more than 8 weeks in osteoclast inhibitor-treated patients with ii) no history of irradiation therapy to the jaws. ONJ of this type following the use of bisphosphonates was first described in 2003 [53]. And in 2008, the so-called 'non-exposed variant of jaw osteonecrosis' was first reported [54], which in the absence of frank bone exposure is characterized by the following clinical features: otherwise unexplained jawbone pain, fistula tracts, loose teeth, swelling, and, in advanced cases, pathologic fracture of the mandible [54, 55].

As risk conditions for the development of ONJ in cancer patients treated with antiresorptive therapies, local factors such as dentures, poor oral hygiene, and preceding dental procedures such as tooth extractions have been identified [23]. Additionally, systemic factors like smoking, diabetes, anemia, renal insufficiency, and use of glucocorticoids, chemotherapeutic and anti-angiogenic agents have been implicated. The risk of ONJ seems to be also enhanced upon longer duration and increased doses of antiresorptive drug therapy [55].

In large meta-analyses of comparative trials in metastatic breast cancer patients, within 2 years of treatment no statistically significant difference in the incidence of ONJ could be detected between denosumab (2.0\% of cases) and zoledronate $(1.4 \%)$ [41, 55-57]. Among nitrogen-containing bisphosphonates, there may be a higher risk of ONJ with zoledronate compared with pamidronate or ibandronate [12].

\section{Management}

Preventive strategies may reduce the incidence of osteoclast inhibitor-associated ONJ [12, 21, 55, 58-60]. These strategies comprise meticulous examination of the oral cavity and completion of any necessary preventive dentistry prior to treatment. While on osteoclast inhibitors, patients should maintain optimal oral hygiene and, if feasible, avoid invasive dental procedures, e.g., implant or periodontal surgery. If invasive dental surgery is required, it seems prudent, although not yet proved formally, to cease therapy prior to the procedure and to resume treatment not until completion of wound healing.

In the case of manifest ONJ, depending on the stage of disease $[23,55,58-60]$, treatment modalities include pain control often requiring opioid medications, antimicrobial mouth rinses $(0.2 \%$ chlorhexidine digluconate), systemic antibiotics according to microbiologic testing (preferably a broad-spectrum penicillin plus metronidazole), and nutritional support when needed. In advanced-stage ONJ with exposed bone, sur- 
gical treatment options such as bone debridement, curettage, sequestrectomy, or resection may be indispensable. Generally, discontinuation of the antiresorptive medication is advised for at least a 1-month postoperative period.

\section{Choice of Adequate Osteoclast Inhibitor}

In breast cancer metastatic to bone, clinical trials have shown superiority of denosumab over bisphosphonates for the prevention of SREs (primary endpoint: time to first onstudy SRE, secondary endpoint: time to first and subsequent (multiple) on-study SREs [20]). Consequently, denosumab may be useful in patients not sufficiently responding to bisphosphonates, although in this matter the definite scientific evidence is still pending.

In toxicity profiles, denosumab lacks nephrotoxicity and induces acute-phase reactions at a markedly lower rate compared to bisphosphonates. On the other hand, hypocalcemia is more common with denosumab. Rates of ONJ, however, occur similarly frequently with both agents. Therefore, it seems logical that in patients with modest degrees of renal impairment, denosumab may be favored. The same holds true for patients with severe renal dysfunction (creatinine clearance $<30 \mathrm{ml} / \mathrm{min}$ ), where zoledronate is contraindicated. In the latter cases, however, the risk of enhanced denosumabinduced hypocalcemia needs careful attention.

Eventually, as a point of patient convenience, the subcutaneous administration of denosumab may be preferable to the intravenous route of zoledronic acid.

\section{Conclusion}

Bisphosphonates and denosumab, notwithstanding their beneficial effects in bone-metastatic breast cancer, are associated with potential adverse events which occur dependent on dose and frequency of administration. These untoward effects of osteoclast inhibitors may add to the already existing symptom burden for patients. Therefore, to alleviate this burden, an appropriate management is required including the following preventive measures:

i) Acute-phase reactions have been linked particularly to bisphosphonates. Consequently, if these problems predominate, switching to denosumab is recommended.

ii) Relating to the risk of nephrotoxicity, serum creatinine level should be checked prior to each dose of zoledronic acid to allow for appropriate dosing modifications or termination of medication. Patients with severe renal dysfunction (creatinine clearance $<30 \mathrm{ml} / \mathrm{min}$ ) should not be treated with zoledronate at all. Generally, adequate hydration is to be maintained.

iii) Hypocalcemia appears to occur more often with denosumab than with zoledronic acid. In any case, however, serum calcium and vitamin D3 levels should be monitored before the first drug administration and all subsequent therapies. All patients treated with osteoclast inhibitors should be given supplemental calcium $(1,000 \mathrm{mg} /$ day orally) plus vitamin D (calcitriol, $0.25 \mu \mathrm{g} /$ day orally) unless they are hypercalcemic.

iv) To reduce the risk of ONJ, meticulous examinations of the oral cavity should be performed prior to and during treatment. Likewise, any necessary preventive dentistry should be completed before therapy. While on osteoclast inhibitors, patients should maintain optimal oral hygiene and avoid invasive dental procedures. Whenever ONJ is diagnosed, the antiresorptive therapy should be discontinued and a specialized center consulted.

\section{Disclosure Statement}

FS has received honoraria from Roche, Novartis, AstraZeneca, and Amgen. FS has been a consultant for Roche, Novartis, and Amgen. CD has received honoraria from Roche.

\section{References}

1 Coleman RE: Metastatic bone disease: clinical features, pathophysiology and treatment strategies. Cancer Treat Rev 2001;27:165-176.

2 Coleman RE: Clinical features of metastatic bone disease and risk of skeletal morbidity. Clin Cancer Res 2006;12:6243s-6249s.

3 Kinnane N: Burden of bone disease. Eur J Oncol Nurs 2007;11(suppl 2):528-531.

4 Pockett RD, Castellano D, McEwan P, et al.: The hospital burden of disease associated with bone metastases and skeletal-related events in patients with breast cancer, lung cancer, or prostate cancer in Spain. Eur J Cancer Care 2010;19:755-760.
5 Sathiakumar N, Delzell E, Morrisey MA, et al.: Mortality following bone metastasis and skeletalrelated events among men with prostate cancer: a population-based analysis of US Medicare beneficiaries, 1999-2006. Prostate Cancer Prostatic Dis 2011;14:177-183.

6 Snedecor SJ, Carter JA, Kaura S, Botteman MF: Denosumab versus zoledronic acid for treatment of bone metastases in men with castration-resistant prostate cancer: a cost-effectiveness analysis. J Med Econ 2013;16:19-29.

7 Smith HS: Painful osseous metastases. Pain Physician 2011;14:E373-E403.

8 Hird A, Zhang L, Holt T, et al.: Dexamethasone for the prophylaxis of radiation-induced pain flare after palliative radiotherapy for symptomatic bone metastases: a phase II study. Clin Oncol 2009;21:329-335.
9 Gnant M, Balic M, Petru E, et al.: Treatment of bone metastases in patients with advanced breast cancer. Breast Care 2012;7:92-98.

10 Ford JA, Jones R, Elders A, et al.: Denosumab for treatment of bone metastases secondary to solid tumours: systematic review and network metaanalysis. Eur J Cancer 2013;49:416-430.

11 Prommer EE: Toxicity of bisphosphonates. J Palliat Med 2009;12:1061-1065.

12 Diel IJ: Bisphosphonates in breast cancer patients with bone metastases. Breast Care 2010;5:306-311.

13 Rogers MJ, Watts DJ, Russell RG: Overview of bisphosphonates. Cancer 1997;80:1652-1660.

14 Boyce BF, Xing L: Functions of RANKL/RANK/ OPG in bone modeling and remodeling. Arch Biochem Biophys 2008;473:139-146. 
15 Anandarajah AP, Schwarz EM: Anti-RANKL therapy for inflammatory bone disorders: mechanisms and potential clinical applications. J Cell Biochem 2006;97:226-232.

16 Diel IJ: Bone metastasis in breast cancer. Breast Care 2012;7:90-91.

17 Hagiwara M, Delea TE, Cong Z, Chung K: Utilization of intravenous bisphosphonates in patients with bone metastases secondary to breast, lung, or prostate cancer. Support Care Cancer 2014;22:103-113.

18 Prommer E: Palliative oncology: denosumab. Am J Hosp Palliat Care 2014; Epub ahead of print.

19 Candelaria-Quintana D, Dayao ZR, Royce ME: The role of antiresorptive therapies in improving patient care in early and metastatic breast cancer. Breast Cancer Res Treat 2012;132:355-363.

20 Stopeck AT, Lipton A, Body JJ, et al.: Denosumab compared with zoledronic acid for the treatment of bone metastases in patients with advanced breast cancer: a randomized, double-blind study. J Clin Oncol 2010;28:5132-5139.

21 Anghel R, Bachmann A, Beksac M, et al.; American Society of Clinical Oncology; National Comprehensive Cancer Network: Expert opinion 2011 on the use of new anti-resorptive agents in the prevention of skeletal-related events in metastatic bone disease. Wien Klin Wochenschr 2013;125:439-447.

22 Fizazi K, Carducci M, Smith M, et al.: Denosumab versus zoledronic acid for treatment of bone metastases in men with castration-resistant prostate cancer: a randomised, double-blind study. Lancet 2011;377:813-822.

-23 Gartrell BA, Coleman RE, Fizazi K, et al.: Toxicities following treatment with bisphosphonates and receptor activator of nuclear factor-kB ligand inhibitors in patients with advanced prostate cancer. Eur Urol 2014;65:278-286.

24 Tanvetyanon T, Stiff PJ: Management of the adverse effects associated with intravenous bisphosphonates. Ann Oncol 2006;17:897-907.

$\checkmark 25$ Fraunfelder FW, Fraunfelder FT: Adverse ocular drug reactions recently identified by the National Registry of Drug-Induced Ocular Side Effects. Ophthalmology 2004;111:1275-1279.

26 Moore MM, Beith JM: Acute unilateral anterior uveitis and scleritis following a single infusion of zoledronate for metastatic breast cancer. Med J Aust 2008;188:370-371.

27 Lenart BA, Lorich DG, Lane JM: Atypical fractures of the femoral diaphysis in postmenopausal women taking alendronate. N Engl J Med 2008; 358:1304-1306.

-28 Saad F, Gleason DM, Murray R, et al.: A randomized, placebo-controlled trial of zoledronic acid in patients with hormone-refractory metastatic prostate carcinoma. J Natl Cancer Inst 2002;94: 1458-1468.

29 Smith MR, Egerdie B, Hernández Toriz N, et al.: Denosumab in men receiving androgen-deprivation therapy for prostate cancer. N Engl J Med 2009;361:745-755.
30 Smith MR, Saad F, Coleman R, et al.: Denosumab and bone-metastasis-free survival in men with castration-resistant prostate cancer: results of a phase 3, randomised, placebo-controlled trial. Lancet 2012;379:39-46.

31 Thiebaud D, Sauty A, Burckhardt P, et al.: An in vitro and in vivo study of cytokines in the acutephase response associated with bisphosphonates. Calcif Tissue Int 1997:61:386-392.

32 Wark JD, Bensen W, Recknor C, et al.: Treatment with acetaminophen/paracetamol or ibuprofen alleviates post-dose symptoms related to intravenous infusion with zoledronic acid $5 \mathrm{mg}$. Osteoporos Int 2012;23:503-512.

33 Chen T, Berenson J, Vescio R, et al.: Pharmacokinetics and pharmacodynamics of zoledronic acid in cancer patients with bone metastases. J Clin Pharmacol 2002;42:1228-1236.

34 Weiss HM, Pfaar U, Schweitzer A, et al.: Biodistribution and plasma protein binding of zoledronic acid. Drug Metab Dispos 2008;36:2043-2049.

35 Perazella MA, Markowitz GS: Bisphosphonate nephrotoxicity. Kidney Int 2008;74:1385-1393.

36 Edwards BJ, Usmani S, Raisch DW, et al.: Acute kidney injury and bisphosphonate use in cancer: a report from the research on adverse drug events and reports (RADAR) project. J Oncol Pract 2013; 9:101-106.

37 Markowitz GS, Appel GB, Fine PL, et al.: Collapsing focal segmental glomerulosclerosis following treatment with high-dose pamidronate. J Am Soc Nephrol 2001;12:1164-1172.

38 Markowitz GS, Fine PL, Stack JI, et al.: Toxic acute tubular necrosis following treatment with zoledronate (Zometa). Kidney Int 2003;64:281-289.

39 Jackson GH: Renal safety of ibandronate. Oncologist 2005;10(suppl 1):14-18.

40 Diel IJ, Bergner R, Grotz KA: Adverse effects of bisphosphonates: current issues. J Support Oncol 2007:5:475-482.

41 Chang JT, Green L, Beitz J: Renal failure with the use of zoledronic acid. N Engl J Med 2003;349:16761679.

42 Berenson JR, Vescio RA, Rosen LS, et al.: A phase I dose-ranging trial of monthly infusions of zoledronic acid for the treatment of osteolytic bone metastases. Clin Cancer Res 2001;7:478-485.

43 Markowitz GS, Fine PL, D'Agati VD: Nephrotic syndrome after treatment with pamidronate. Am J Kidney Dis 2002:39:1118-1122.

44 Bergner R, Diel IJ, Henrich D, et al.: Differences in nephrotoxicity of intravenous bisphosphonates for the treatment of malignancy-related bone disease. Onkologie 2006;29:534-540.

45 Block GA, Bone HG, Fang L, et al.: A single-dose study of denosumab in patients with various degrees of renal impairment. J Bone Miner Res 2012; 27:1471-1479.

46 Van Poznak CH, Von Roenn JH, Temin S: American society of clinical oncology clinical practice guideline update: recommendations on the role of bone-modifying agents in metastatic breast cancer. J Oncol Pract 2011;7:117-121.
47 Lechner B, DeAngelis C, Jamal N, et al.: The effects of denosumab on calcium profiles in advanced cancer patients with bone metastases. Support Care Cancer 2014;22:1765-1771.

48 Body J-J, Lipton A, Henry D, et al.: Incidence of hypocalcemia among denosumab-treated patients enrolled in three registrational phase 3 trials. IBMS BoneKEy 9, Article number: 193(2012):S24-S25 1; doi:10,1038/bonekey.2012,193.

49 Milat F, Goh S, Gani LU, et al.: Prolonged hypocalcemia following denosumab therapy in metastatic hormone refractory prostate cancer. Bone 2013;55:305-308.

-50 Kreutle V, Blum C, Meier C, et al.: Bisphosphonate induced hypocalcemia - report of six cases and review of the literature. Swiss Med Wkly 2014; 144:w13979.

51 Buonerba C, Caraglia M, Malgieri S, et al.: Calcitriol: a better option than vitamin $\mathrm{D}$ in denosumabtreated patients with kidney failure? Expert Opin Biol Ther 2013;13:149-151.

52 Advisory Task Force on Bisphosphonate-Related Osteonecrosis of the Jaws, American Association of Oral and Maxillofacial Surgeons: American Association of Oral and Maxillofacial Surgeons position paper on bisphosphonate-related osteonecrosis of the jaws. J Oral Maxillofac Surg 2007;65:369-376.

53 Marx RE: Pamidronate (Aredin) and zoledronate (Zometa) induced avascular necrosis of the jaws: a growing epidemic. J Oral Maxillofac Surg 2003;61: 1115-1117.

54 Jungnera L, Gallego L: Nonexposed bisphosphonate-related osteonecrosis of the jaws: another clinical variant? J Oral Maxillofac Surg 2008;66: 1516-1517.

55 Campisi G, Fedele S, Fusco V, et al.: Epidemiology, clinical manifestations, risk reduction and treatment strategies of jaw osteonecrosis in cancer patients exposed to antiresorptive agents. Future Oncol 2014;10:257-275.

56 Van den Wyngaert T, Wouters K, Huizing MT, Vermorken JB: RANK ligand inhibition in bone metastatic cancer and risk of osteonecrosis of the jaw (ONJ): non bis in idem? Support Care Cancer 2011;19:2035-2040.

57 Wang X, Yang KH, Wanyan P, Tian JH: Comparison of the efficacy and safety of denosumab versus bisphosphonates in breast cancer and bone metastases treatment: a meta-analysis of randomized controlled trials. Oncol Lett 2014;7:1997-2002.

58 Epstein MS, Epstein JB, Ephros HD: The effects of osteoclast modifiers on the oral cavity: a review for prescribers. Curr Opin Support Palliat Care 2012;6:337-341.

59 Uyanne J, Calhoun CC, Le AD: Antiresorptive drug-related osteonecrosis of the jaw. Dent Clin North Am 2014;58:369-384.

60 De Iuliis F, Taglieri L, Amoroso L, et al.: Prevention of osteonecrosis of the jaw in patients with bone metastases treated with bisphosphonates. Anticancer Res 2014;34:2477-2480. 